\title{
Bilateral Control of a Nonlinear Pneumatic Teleoperation System with Solenoid Valves
}

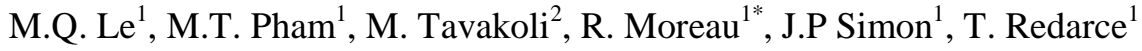 \\ ${ }^{1}$ Laboratoire Ampère, UMR CNRS 5005, \\ Université de Lyon, INSA-Lyon, F-69621 Villeurbanne Cedex, France \\ ${ }^{2}$ Department of Electrical and Computer Engineering, University of Alberta, Canada \\ *Corresponding author's e-mail: richard.moreau@insa-lyon.fr
}

\begin{abstract}
In past research on the control of pneumatic actuators, typically proportional servovalves have been used for achieving high-performance control of the mass flow rate. In this paper, we instead use fast-switching on/off valves due to their distinct advantages in terms of low cost and small size. Accurate control of pneumatic actuators with on/off solenoid valves is a challenge since the system dynamics is both discrete-input and highly nonlinear. In this paper, we apply a hybrid control algorithm to a pneumatic actuator with on/off valves. Such a control approach is developed for choosing the best control vector at each sample time to track the reference state (i.e., desired force) in the inner force control loop within a bilateral teleoperation system. Experimental results show that good teleoperation transparency is achieved despite all the obstacles such as discrete-input and nonlinear behavior of the pneumatic-actuated teleoperation system.
\end{abstract}

Index terms-Pneumatic actuators, on/off solenoid valve, hybrid control, haptic teleoperation, bilateral control, transparency.

\section{INTRODUCTION}

From its early use in the remote manipulation of radioactive materials, the application of teleoperation has expanded to include manipulation at different scales and in virtual worlds [1]. Teleoperation systems are useful in remote or hazardous operations such as space and undersea explorations and in delicate operations such as micro-surgery and micro-assembly.

In a bilateral teleoperation system, apart from the basic requirement of stability, there are primarily two control design goals for ensuring a close coupling between the human operator and the environment. The first goal is that the slave manipulator tracks the position of the master manipulator, and the second goal is that the environment force acting on the slave is accurately displayed to the master. These goals result in transparency of the teleoperation system, meaning that through the master manipulator, the operator feels as if he/she is directly operating on the remote environment [2].

The actuators most used today in haptic systems are direct-current electrical motors. They are easy to install, quiet and relatively simple to control. However, their reduction mechanisms can introduce backlash and high inertia, which are undesirable in haptic applications. In this study, we investigate the use of electro-pneumatic actuators in a teleoperation 
system. Compared to the electrical actuators, pneumatic actuators have higher force-to-mass ratio and can generate larger force without any reduction mechanism. Moreover they are inert to magnetic fields, which is crucial in certain applications such as robot-assisted surgery under MRI (Magnetic Resonance Imaging) guidance [3], [4].

Due to the above advantages, pneumatic actuators have found use in new applications such as tele-robotics in recent years. For instance, Tadano and Kawashima [5] propose a forceps manipulator for a surgical master-slave system, which is able to estimate external forces without using any force sensor. Pneumatic muscle actuators have been also recently used in the teleoperation system [6]. They are compact and have high power/weight density, but they are difficult to control and require an accurate experimental characterization.

All of the prior works used proportional servo-valves in pneumatically actuated systems because they allow achieving high performances in various position or force control tasks. However they are typically expensive due to requiring highprecision manufacturing. Therefore, in this paper, fast-switching on/off valves are used due to their advantages in terms of low cost and small size. Thanks to the breakthroughs in valve technology such as leak reduction, miniaturized of mechanical elements and fast electronic components, the solenoid valves now are faster and more accurate than the former valves. One of the objectives of this paper is to show that good transparency in teleoperation can be obtained with these inexpensive components.

The traditional method for controlling a system with a solenoid valve is Pulse Width Modulation (PWM) [7-9]. A main problem with PWM control is the chattering that is caused by the high-frequency switching of the valve [10]. Chattering can drastically reduce the valve's lifetime and generate noises possibly disturbing for certain applications.

To overcome the drawbacks of PWM-based control of solenoid valves, this paper presents a new control method inspired by the hybrid theory recently developed for asynchronous/synchronous electrical motors control [11-13]. This approach is used in a switching-based hybrid system, which includes continuous actuators and a discrete controller with a finite number of states. In the case of alternating-current (AC) motor drives, contrary to conventional vector control such as proportional-integral control in which the inverter model is not taken into account by the controller, hybrid control considers the state of the inverter as a control variable. Therefore, it allows obtaining faster torque dynamics than the vector-control algorithm. Our contribution in this paper consists of applying the previously-developed hybrid algorithm to the pneumatic master-slave system actuated by fast switching on/off valves. Four-channel $(4 \mathrm{CH})$ bilateral teleoperation architecture is used in order to test the efficiency of the proposed control laws. The reason for this is that the four-channel method is the most general teleoperation control architecture compared to position error based (PEB), direct force reflection (DFR), and shared 
compliance control (SCC) methods and is one that can achieve superior transparency [14], [15]. Finally, an analysis of the controller parameters is carried out to achieve satisfactory performance in terms of teleoperation transparency.

For the sake of simplicity, the master and slave actuators are considered to be identical in this study. In the experiments, the master and the slave are one degree of freedom (DOF) pneumatic manipulators. It should be noted that this paper does not deal with the presence of time delay in the teleoperation system's communication channel - for time delay compensation in haptic teleoperation, the readers may refer to [16], [17]. Such delay is commonly presented in remote teleoperation systems, but is generally not significant in local teleoperation systems.

The structure of this paper is as follows. First, the modeling of the pneumatic manipulator composed of a cylinder and four solenoid valves is presented in Section II. Section III describes the hybrid principle which is implemented in a fourchannel bilateral control system. Section IV presents experimental results that validate the proposed theories. Finally, concluding remarks appear in Section V.

\section{MODEL OF THE PNEUMATIC ACTUATOR}

As mentioned above, the master and the slave manipulators are identical, thus only one pneumatic robot is presented in this section. To describe the air flow dynamics in a cylinder, we assume that [18]

- Air is a perfect gas and its kinetic energy is negligible in the chamber,

- The pressure and the temperature are homogeneous in each chamber,

- The evolution of the gas in each cylinder chamber is polytropic,

- The temperature variation in chambers is negligible with respect to the supply temperature,

- The mass flow rate leakages are negligible, and

- The supply and exhaust pressures are constant.

A schematic of a 1-DOF pneumatic actuation system is shown in Fig. 1. The device consists of a pneumatic cylinder, four solenoid valves, a position sensor and two pressure sensors. Each chamber is connected to two solenoid valves. Valves 1 and 4 are connected to the supply pressure while valves 2 and 3 are connected to the atmosphere pressure. The choice of four valves rather than two valves allows us to increase the degrees of freedom in the choice of the control vector and the resulting behavior of the closed-loop system.

The behavior of the pressure inside each chamber of the cylinder can be expressed as [19] 


$$
\left\{\begin{array}{l}
\frac{d P_{p}}{d t}=\frac{\gamma r T_{a}}{V_{p}(y)}\left(q_{p}\left(U_{1}, U_{2}, P_{p}\right)-\frac{P_{p}}{r T_{a}} S_{p} \dot{y}\right) \\
\frac{d P_{n}}{d t}=\frac{\gamma r T_{a}}{V_{n}(y)}\left(q_{n}\left(U_{3}, U_{4}, P_{n}\right)+\frac{P_{n}}{r T_{a}} S_{n} \dot{y}\right)
\end{array}\right.
$$

where $U_{1}, U_{2}, U_{3}$ and $U_{4}$ are the control voltages of the valve 1 , valve 2 , valve 3 , and valve $4, y$ and $\dot{y}$ are the position (m) and velocity $(\mathrm{m} / \mathrm{s})$ of the piston, $P_{p}$ and $P_{n}$ are the pressures inside chambers $\mathrm{p}$ and $\mathrm{n}(\mathrm{Pa}), V_{p}$ and $V_{n}$ are the volumes of chambers $\mathrm{p}$ and $\mathrm{n}\left(\mathrm{m}^{3}\right), S_{p}$ and $S_{n}$ are the piston cylinder area of chambers $\mathrm{p}$ and $\mathrm{n}\left(\mathrm{m}^{2}\right), q_{p}$ and $q_{n}$ are the mass flow rates in chambers $\mathrm{p}$ and $\mathrm{n}(\mathrm{kg} / \mathrm{s}), T_{a}$ is the temperature of the supply air $(\mathrm{K}), r$ is the perfect gas constant $(\mathrm{J} / \mathrm{kg} / \mathrm{K})$ and $\gamma$ is the polytropic constant.

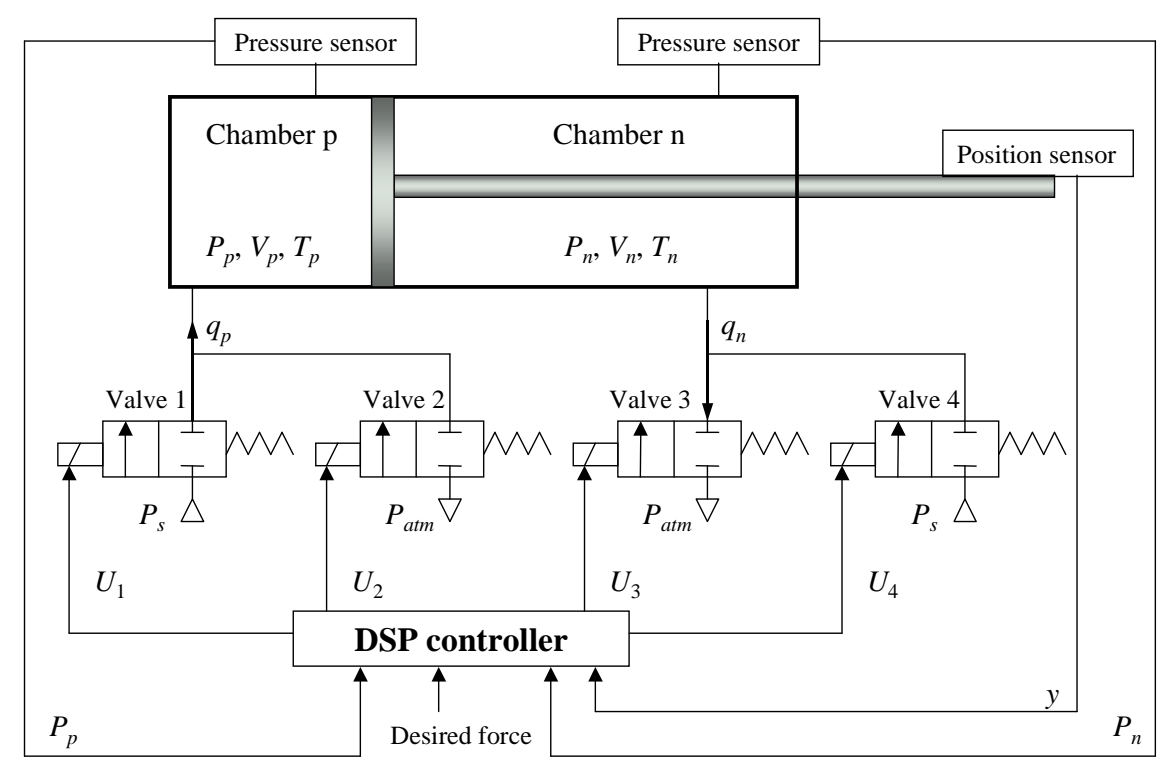

Fig. 1. Electro-pneumatic force control system with four on/off valves

The mass flow rate characteristic of the on/off valves can be expressed as a function of the discrete control voltages and the pressures:

$$
\begin{aligned}
& q_{p}\left(U_{1}, U_{2}, P_{p}\right)= \begin{cases}q_{m}\left(P_{s}, P_{p}\right) & \text { for } U_{1}=1 \text { and } U_{2}=0 \text { (chamber p fills) } \\
0 & \text { for } U_{1}=0 \text { and } U_{2}=0 \text { (chamber p closed ) } \\
-q_{m}\left(P_{p}, P_{a t m}\right) & \text { for } U_{1}=0 \text { and } U_{2}=1 \text { (chamber p exhauts) }\end{cases} \\
& q_{n}\left(U_{3}, U_{4}, P_{n}\right)= \begin{cases}q_{m}\left(P_{s}, P_{p}\right) & \text { for } U_{3}=0 \text { and } U_{4}=1 \text { (chamber } \mathrm{n} \text { fills) } \\
0 & \text { for } \left.U_{3}=0 \text { and } U_{4}=0 \text { (chamber } \mathrm{n} \text { closed }\right) \\
-q_{m}\left(P_{p}, P_{a t m}\right) & \text { for } U_{3}=1 \text { and } U_{4}=0 \text { (chamber } \mathrm{n} \text { exhauts) }\end{cases}
\end{aligned}
$$

where $P_{s}$ and $P_{a t m}$ are the pressures of the supply air and the atmosphere. The ' 0 ' state of the input voltage corresponds to a closed valve and the ' 1 ' state corresponds to an open valve. In the above, the cases of $U_{1}=U_{2}=1$ and $U_{3}=U_{4}=1$ have 
been prohibited to avoid a bypass of the valves. The function $q_{m}$ in (2) is given by a standard expression for the mass flow rate through an orifice of constant area, which depends only on the upstream and downstream pressures [20]:

$$
q_{m}\left(P_{u p}, P_{d o w n}\right)=\left\{\begin{array}{l}
C P_{u p} \sqrt{T_{a t m} / T_{u p}} \text { if } P_{\text {down }} / P_{u p} \leq C_{r} \text { (sonic) } \\
C P_{u p} \sqrt{T_{a t m} / T_{u p}} \sqrt{1-\left(\frac{P_{\text {down }} / P_{u p}-C_{r}}{1-C_{r}}\right)^{2}} \text { otherwise (subsonic) }
\end{array}\right.
$$

where $C_{r}$ is the critical pressure ratio and $C$ is the sonic conductance, $\left(C_{r}=0.433, C=17.10^{-5} \mathrm{Nl} / \mathrm{mn} / \mathrm{Pa}\right.$ according to the ISO 6358 standard), $P_{u p}$ and $P_{\text {down }}$ are respectively the absolute upstream and downstream stagnation pressures of the valve (Pa), $T_{a t m}$ is the atmosphere temperature, and $T_{u p}$ is the upstream stagnation temperature. Thereafter, the pair $\left(C, C_{r}\right)$ is supposed to be constant and identical for all solenoid valves.

The dynamics of the piston and the load are

$$
M \ddot{y}=S_{p} P_{p}-S_{n} P_{n}-b \dot{y}-F_{s t}
$$

where $b$ is the viscous friction coefficient (N.s/m), $M$ is the mass $(\mathrm{kg})$, and $F_{s t}$ is the stiction force. In our case, the stiction force is considered to be negligible since the pneumatic actuator used in experiment is a frictionless cylinder (Airpel model).

\section{BILATERAl CONTROL OF A PNEUMATIC TELEOPERATION SYSTEM}

As it will be seen later, two inner force control loops exist within a pneumatically-driven bilateral teleoperation control system. In the following, we first develop a hybrid control based predictive approach to track the desired force in each inner loop. Then, we incorporate two such hybrid controllers into the four-channel bilateral control architecture and discuss the transparency in terms of position and force tracking of the entire closed-loop haptic teleoperation system.

\section{A. Hybrid control of a single pneumatic manipulator}

\section{1) Hybrid control principle}

Hybrid control uses a hybrid model where the continuous states of a continuous system depend on the configuration of the energy modulator (i.e., the solenoid valves)

$$
\underline{\dot{X}}(t)=f(\underline{X}(t), \underline{u}(t))
$$

with the state vector $\underline{X}=\left(x_{1}, x_{2}, \ldots, x_{\mathrm{m}}\right) \in \mathrm{R}^{\mathrm{m}}$. Here, $f$ is the dynamic function governing the state-space model of the continuous-time system and $\underline{u}$ is a control vector that has a discrete nature and can correspond to any of the $N$ possible configurations of the energy modular. In other words,

$$
\underline{u} \in\left\{\underline{u}_{1}, \underline{u}_{2}, \ldots, \underline{u}_{N}\right\}_{N \geq 2}
$$


where each vector member of the above set represents a unique configuration for the outputs of the solenoid valves in the pneumatic system.

For a small sampling period $T$, the dynamic model (5) can be approximated by a discrete model using the forwarddifference method:

$$
\underline{X}((k+1) T) \approx \underline{X}(k T)+f(\underline{X}(k T), \underline{u}(k T)) \cdot T
$$

The full state $\underline{X}(k T)$ is assumed to be measured at the sample time $k T$. The state at the sample time $(k+1) T$, denoted by $\underline{X}_{\mathrm{j}}((k+1) T)$, resulting from the energy modulator's $j$-th configuration, $1 \leq j \leq N$, can be calculated by (7). The $N$ directions $\underline{d}_{\mathrm{j}}$ in the state space are defined as

$$
\underline{d}_{j}=\underline{X}_{j}((k+1) T)-\underline{X}(k T)
$$

For a given reference state $\underline{X}_{\text {ref }}$, the hybrid control calculates the $N$ possible directions $\underline{d}_{j}$. Then, an optimal control among the $N$ configurations is chosen in order to track this reference state in the state space as closely as possible.

For more details about the hybrid control, the reader can refer to [11], [21].

\section{2) Application to a pneumatic system}

Because the control signals of the master and slave manipulators in an impedance-type teleoperation system are force signals [22], the hybrid algorithm presented in Section III.A.1 is applied to a force tracking problem. The bilateral control of the teleoperation system will be detailed in Section III.B. For the system of Fig. 1 and the force tracking problem, the pressures in chambers $\mathrm{p}$ and $\mathrm{n}$ can be used to form the state vector $X(t)=\left(P_{n} P_{p}\right)^{\mathrm{T}}$. At any given time, each valve may show three different input-output behaviors (pressure admission, closed, and pressure exhaust) as shown in (2).

Since the cases $U_{1}=U_{2}=1$ and $U_{3}=U_{4}=1$ have been prohibited to avoid a bypass of the valves, this leads to nine different control vectors $\underline{u}_{1}$ to $\underline{u}_{9}$, as shown in Table I. Note that the first control configuration in Table I is used to conserve energy and eliminate chattering at steady state.

TABLE I

\begin{tabular}{|c|c|c|c|c|c|c|c|c|c|}
\hline$U_{1}$ & 0 & 1 & 0 & 0 & 0 & 1 & 0 & 0 & 1 \\
\hline$U_{3}$ & 0 & 0 & 0 & 1 & 0 & 1 & 0 & 1 & 0 \\
\hline$U_{4}$ & 0 & 0 & 0 & 0 & 1 & 0 & 1 & 0 & 1 \\
\hline Chamber $\mathrm{n}$ & closed & closed & closed & exhausts & fills & exhausts & fills & exhausts & fills \\
\hline
\end{tabular}

NINE DISCRETE POSSIBLE CONTROL CONFIGURATIONS 
As discussed before, knowing the pressures in both chambers at the sample time $k T$, the objective is to estimate the evolution of the pressures at the next sample time $(k+1) T$ in chambers $\mathrm{p}$ and $\mathrm{n}$ for the nine possible control configurations (Table I), and then choose the best control configuration for reaching the desired force. Assuming the variations of the pressures during a sampling time are small, the derivatives of the pressures can be discretized similar to (7) as

$$
\left\{\begin{array}{l}
\left.P_{p}((k+1) T) \approx \frac{d}{d t}\left(P_{p}(t)\right)\right|_{t=k T} T+P_{p}(k T) \\
\left.P_{n}((k+1) T) \approx \frac{d}{d t}\left(P_{n}(t)\right)\right|_{t=k T} T+P_{n}(k T)
\end{array}\right.
$$

where the derivatives of the pressures $d P_{p} / d t$ and $d P_{n} / d t$ are calculated based on (1)-(3) and are functions of $P_{p}, P_{n}, y$ and $\dot{y}$. At each sample time, $P_{p}, P_{n}$ and $y$ are measured by sensors, while $\dot{y}$ is estimated by numerical derivation of the position measurement $y$. Thus, for each of the nine control configurations, the algorithm calculates $P_{p}((k+1) T)$ and $P_{n}((k+1) T)$ based on (9). Consequently, the nine directions $\underline{d}_{1}$ to $\underline{d}_{9}$ found from (8) define the set of reachable points at time $(k+1) T$ in the state space (See Fig. 2).

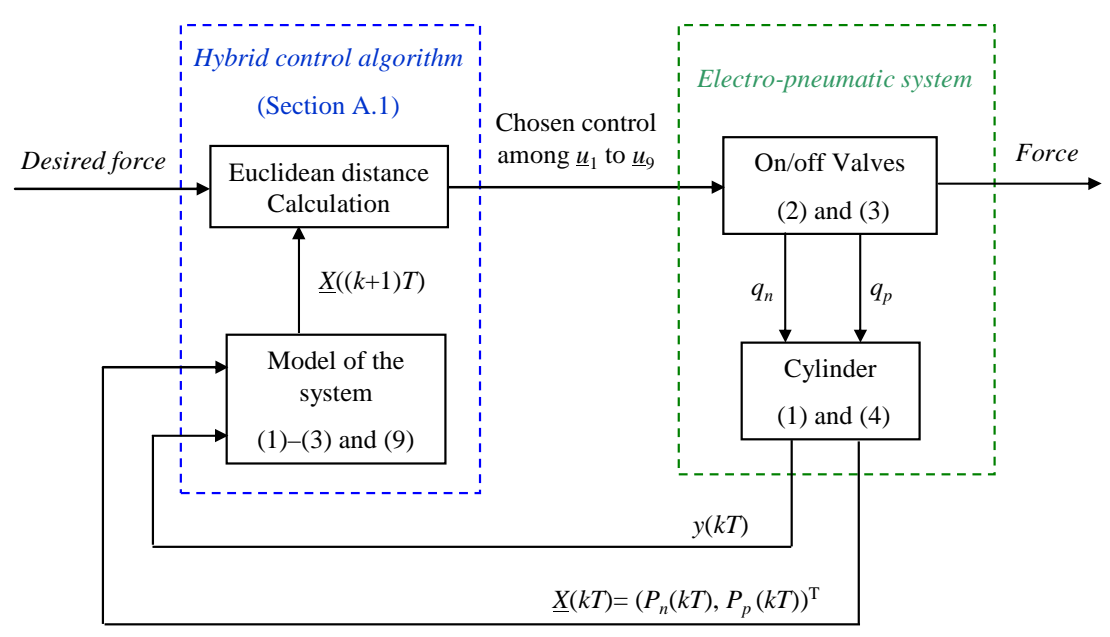

Fig. 2. Block diagram of the hybrid control system

Because each cylinder has 2 chambers, thus the dimension of the state space is $m=2$ in a pneumatic manipulator. For a given desired force $F$, the set of target points (i.e., desired states) is defined by a straight line because

$$
P_{p}=\frac{F}{S_{p}}+\frac{P_{n} S_{n}}{S_{p}}+\frac{P_{a t m}\left(S_{p}-S_{n}\right)}{S_{p}}
$$

Knowing the set of target points, the hybrid algorithm selects the optimal configuration that corresponds to the smallest Euclidean distance between the set of the nine reachable points and the set of target points. The choice of the best control 
configuration has to also take into account that the selected target point is restricted to the domain defined by an upper limit $\left(P_{s}\right)$ and a lower limit $\left(P_{a t m}\right)$ on the pressures in the two chambers [23].

\section{B. Bilateral $4 \mathrm{CH}$ control of a pneumatic master-slave teleoperation system}

Fig. 3 depicts the general 4CH bilateral teleoperation architecture proposed by Lawrence [24].

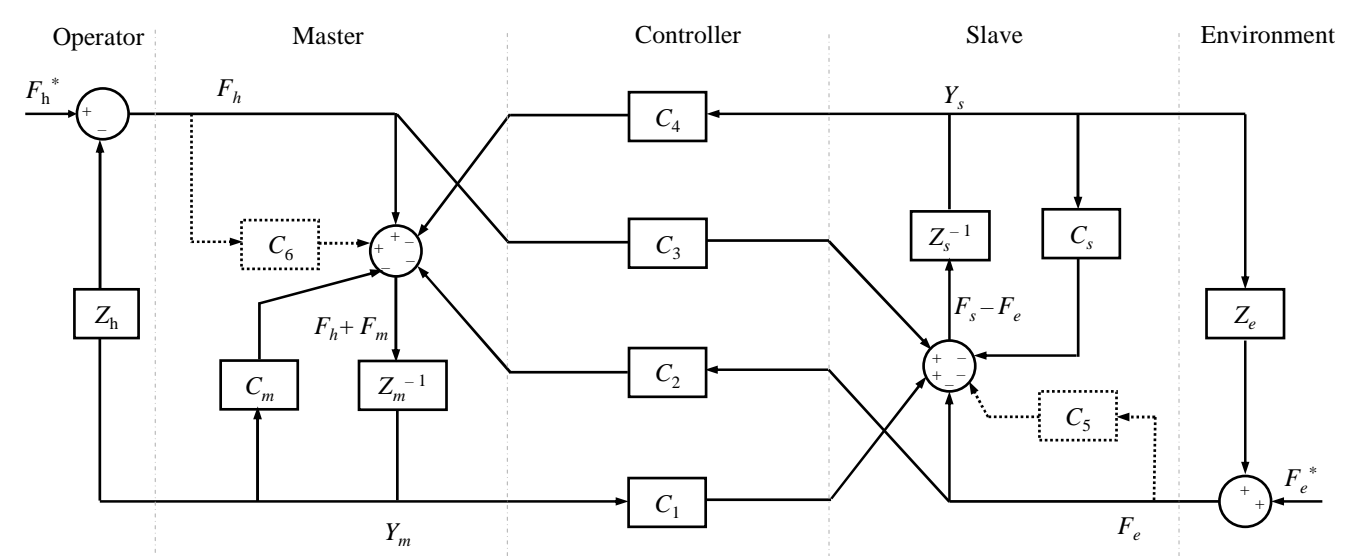

Fig. 3. 4CH bilateral control architecture

In Fig. 3, $Z_{m}$ and $Z_{s}$ denote the master and slave manipulators' linearized dynamics, $Z_{h}$ and $Z_{e}$ denote the dynamic characteristics of the human operator's hand and the environment, $Y_{m}$ and $Y_{s}$ are the master and slave positions, $F_{h}$ and $F_{e}$ are the operator force exerted on the master and the environment force exerted on the slave, $F_{m}$ and $F_{s}$ are the force control signals for the master and slave manipulators, $C_{m}$ and $C_{s}$ denote the local position controllers of the master and the slave sides, $C_{5}$ and $C_{6}$ are local force feedback terms for the master and the slave, $C_{1}$ to $C_{4}$ are position or force controllers embedded in the communication channel, and $F_{h}{ }^{*}$ and $F_{e}{ }^{*}$ are the operator's and the environment's exogenous input forces.

Note that throughout this paper, we use positions instead of velocities as shown in Lawrence's architecture [24]. Generally, when the delay in the communication channel is negligible, the use of position controllers or velocity controllers does not affect the stability of the teleoperation system, thus we opt to use position controllers [14].

In an ideally transparent teleoperation system, the master and the slave positions and forces will match regardless of the operator and environment dynamics:

$$
Y_{m}=Y_{s} \quad, \quad F_{h}=F_{e}
$$

By selecting $C_{1}$ through $C_{6}$ according to

$$
C_{1}=Z_{s}+C_{s} \quad C_{4}=-\left(Z_{m}+C_{m}\right) \quad C_{6}+1=C_{2} \quad C_{5}+1=C_{3}
$$

the perfect transparent condition (16) is fully met. 
According to [25], at low frequencies, near-transparency can be obtained by ignoring the inertial terms in the expressions for $C_{1}$ and $C_{4}$ in which case the original control design (12) is modified to

$$
C_{1}=C_{s} \quad C_{4}=-C_{m} \quad C_{6}+1=C_{2} \quad C_{5}+1=C_{3}
$$

Normally, the position controllers are chosen such that $C_{m} / C_{s}=Z_{m} / Z_{s}$. Since in our experiments the master and the slave robots are identical $Z_{m}=Z_{s}=Z$, we take their controllers to be similar as well:

where $C_{p}$ and $C_{f}$ are the position and force controllers.

$$
C_{s}=C_{m}=C_{p} \quad C_{2}=C_{3}=C_{f}
$$

Thanks to the transparency conditions (13) and the assumption (14), the 4CH bilateral teleoperation architecture in Fig. 3 with eight controller parameters $\left(C_{1}\right.$ to $C_{6}, C_{m}$ and $\left.C_{s}\right)$ has been simplified to only two controllers $\left(C_{p}\right.$ and $\left.C_{f}\right)$, as shown in Fig. 4.

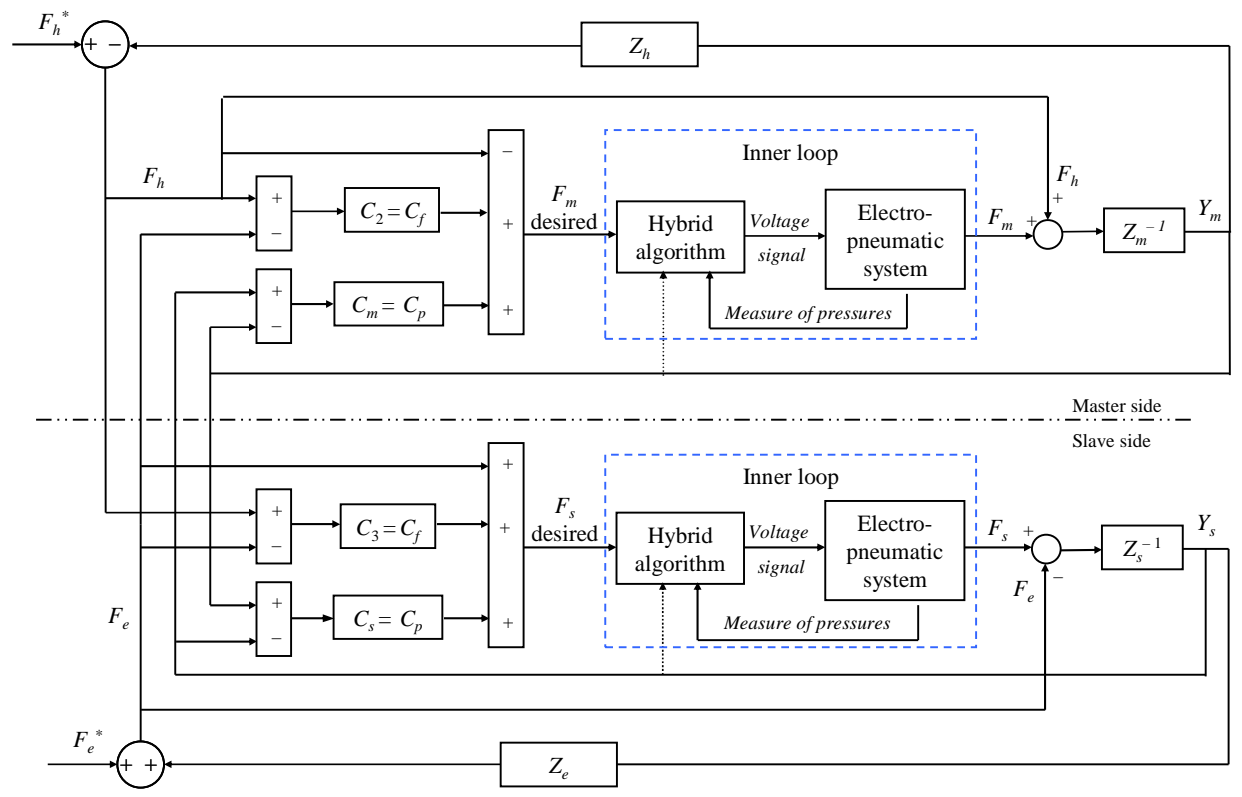

Fig. 4. 4CH bilateral teleoperation block diagram with hybrid control

A main difference between the original diagram in Fig. 3 and the diagram presented in Fig. 4 is that the hybrid algorithm for force control of pneumatic manipulators has been incorporated into Fig. 4. The teleoperation control signals $F_{m}$ and $F_{s}$ correspond to the desired force that is input to the hybrid force control loops shown in Fig. 2. Stability analysis for the closed-loop system is not easy given the discrete-input nature of the pneumatic actuator. However, with good force tracking obtained with the hybrid control, the behavior of the inner loop may be approximated by a unitary transfer function, reducing the block diagram of Fig. 5 to the standard block diagram of Fig. 4. In the standard 4-channel architecture of Fig. 4, the 
passivity (and thus the closed-loop stability) can be established via analysis of the teleoperation system's scattering matrix, which can be shown to have singular values no greater than unity [15].

\section{EXPERIMENTS}

\section{A. Experimental setup}

In this section, experiments with a 1-DOF teleoperation system are reported. As illustrated in Fig. 5, the setup consists of two master and slave identical manipulators. The low-friction cylinders (Airpel model) have a $16 \mathrm{~mm}$ diameter and a $100 \mathrm{~mm}$ stroke. In terms of actuators, the pneumatic cylinder uses four solenoid valves for each manipulator. The pneumatic solenoid valves (Matrix model) used to control the air flow have switching times of approximately $1.3 \mathrm{~ms}$ (opening time) and $0.2 \mathrm{~ms}$ (closing time). In terms of sensors, a low-friction linear variable differential transformer sensor is connected to each cylinder in order to measure the master's and the slave's positions. Also, the end-effector of each manipulator is equipped with a force sensor in order to measure the operator's and the environment's forces. In addition, each cylinder chamber is equipped with a pressure sensor. The system was supplied with air at an absolute pressure of $300 \mathrm{kPa}$.

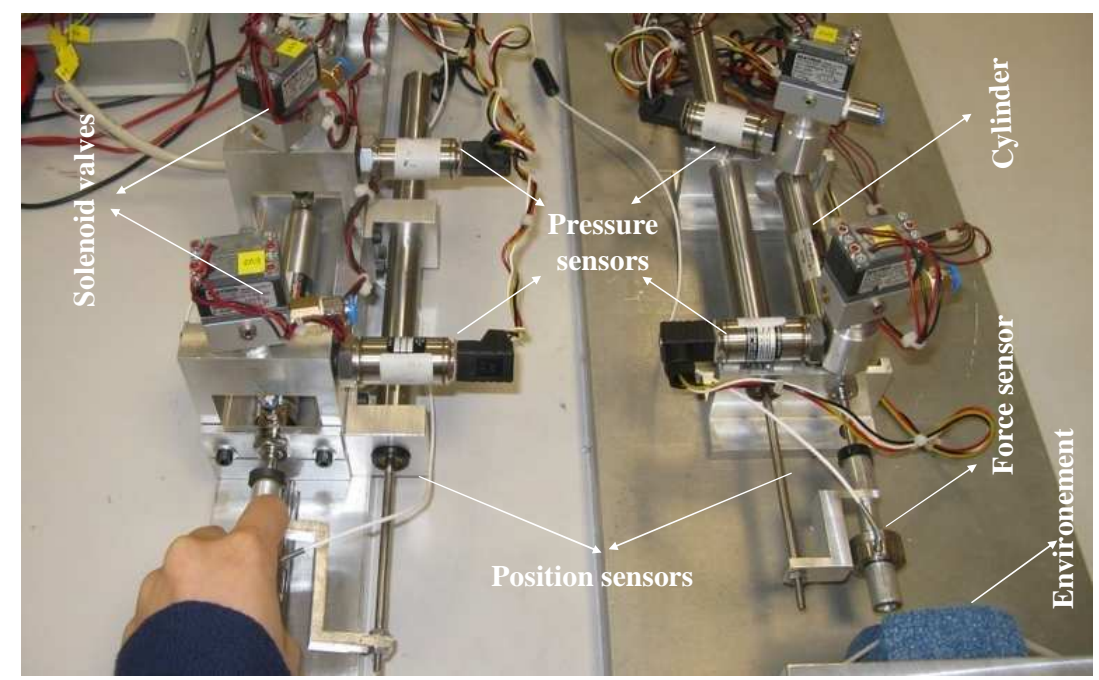

Fig. 5. Pneumatic master-slave teleoperation experimental setup

The controller is implemented using a dSPACE board (DS1104), running at a sampling rate of $500 \mathrm{~Hz}$. This value has been chosen according to the open/close bandwidth of the valves and to guarantee acceptable tracking response. This sampling rate is also higher than the bandwidth above which the human finger cannot distinguish two consecutive force stimuli which is $320 \mathrm{~Hz}$ [26]. 


\section{B. Experimental results}

\section{1) Time domain analysis}

a) Four-channel case

Fig. 6 shows the force and position tracking responses of the $4 \mathrm{CH}$ scheme obtained in the experiments. For the first few seconds, the master is moved back and forth by the user while the slave is in free space. The nonzero values for $F_{h}$, even when the slave is in free space, is mainly due to the uncompensated the mass of the handle between the force sensor and the operator's hand. As it can be seen, in free motion, the slave rapidly tracks the master's motion. Next, the slave makes contact with the environment. The operator pushes against the master leading to different levels of the slave/environment contact forces. The controller ensures a good agreement between the operator/master and the slave/environment forces under contact motion. The environment consists of a sponge with stiffness such as a slight penetration of the slave interface into the environment is possible. However, since this penetration is small for considerable operator forces, we consider this environment to provide a good approximation to a stiff environment; the reason for not using an infinitely-stiff environment (e.g., metal) in our experiments lies in trying to avoid force spikes at the onset of slave/environment contact.

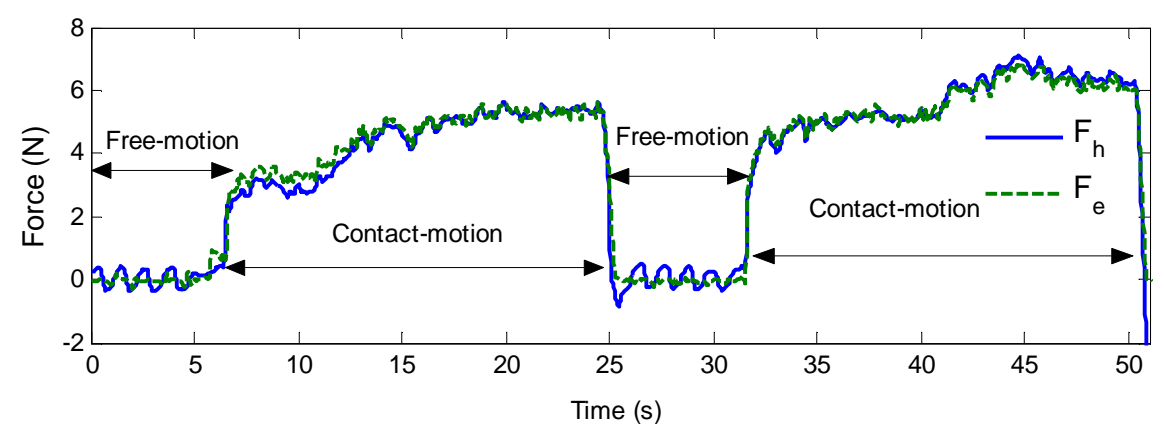

(a). Force tracking

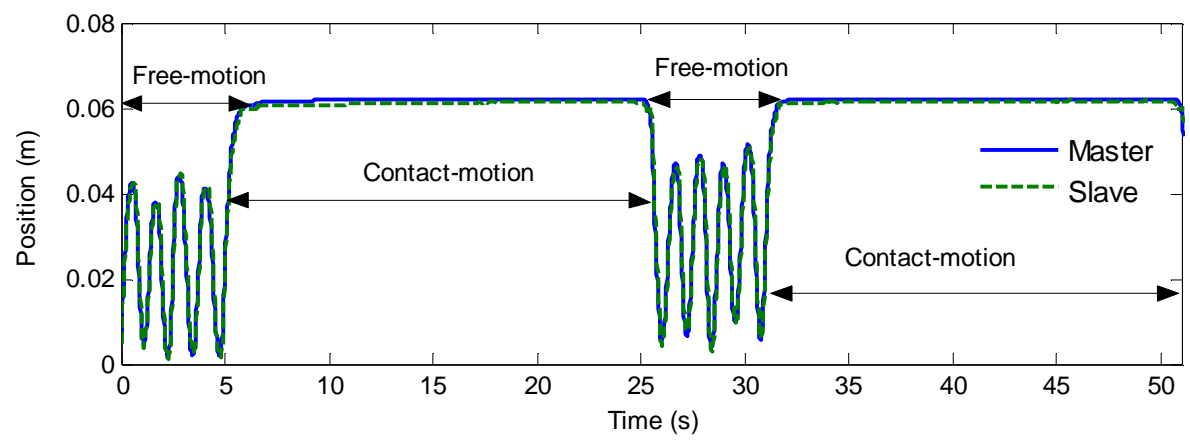

(b). Position tracking

Fig. 6. Transparent performance achieved with the experimental $4 \mathrm{CH}$ teleoperation systems

As mentioned in Section III.B, transparency can be achieved in the four-channel teleoperation system in Fig. 6 subject to the choice of controllers $C_{m}, C_{s}, C_{2}$ and $C_{3}$ in (14) as well as $C_{6}=C_{2}-1$ and $C_{5}=C_{3}-1$ from (13), if the inner force control 
loop is perfect. This condition involving the inner force control loop is verified in Fig. 7, where the force generated by the slave actuator accurately tracks the desired value for the control signal $F_{s}$. A similar result can be shown for the master-side inner force control loop. It is interesting to note that the performance of the inner-loop force control is less accurate in the free-motion case than in the contact-motion case. Indeed, in free motion where the piston position significantly varies and so do the volume chambers, the pressure variation increase according to (1). This leads to decreased accuracy of the pressure approximation in (9). Therefore, the performance of the inner-loop force control deteriorates during free motion. On the other hand, in contact motion where the position is nearly constant, the pressure variation is small, the pressure approximation in (9) is highly accurate, and thus good inner-loop force tracking performance can be achieved (see Fig. 7).
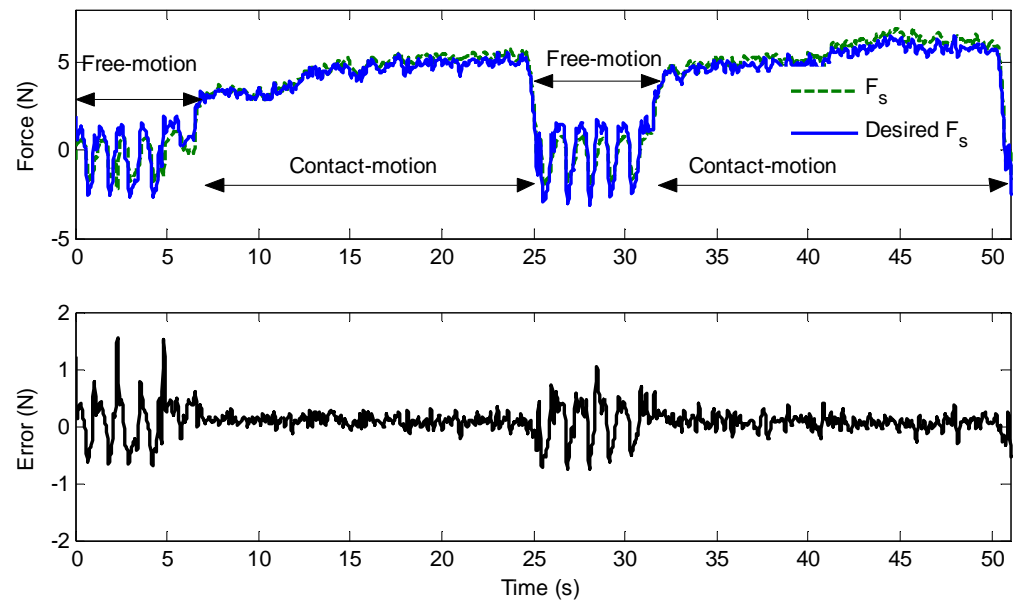

Fig. 7. Performance of the inner force control loop

To better understand the dynamic behavior of the inner force control loop, a spectra analysis is investigated, as illustrated in Fig. 8. It can be seen that the transfer function of the inner loops approaches to the unitary function at low frequencies (less than $2 \mathrm{~Hz}$ ). For faster movements $(e . g$. , at $7 \mathrm{~Hz})$, the response is degraded. In conclusion, the pressure/force prediction is sensitive to the movement bandwidth of the master/slave manipulator. In our experimental validation, the arm movements were slow enough to be able to assume that highly transparent teleoperation system (whose response is shown in Fig. 6) can be obtained through the employed hybrid force control (whose response is shown in Fig. 7). 


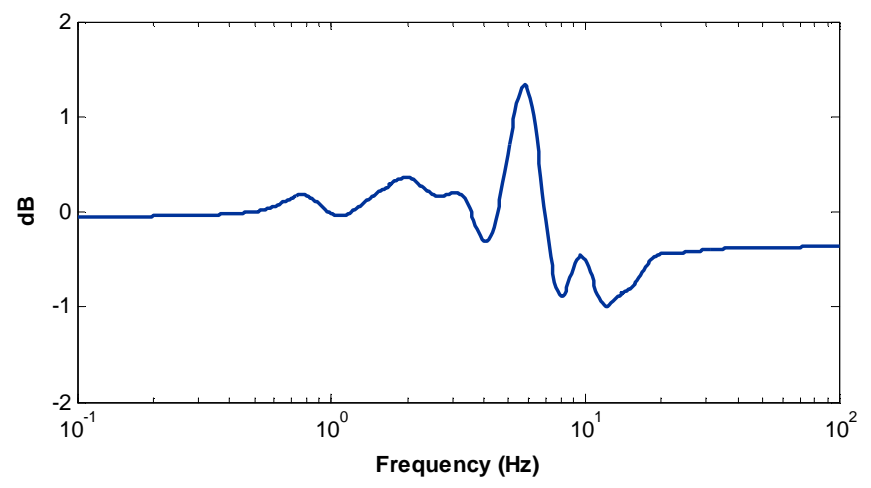

Fig. 8. Frequency analysis of the inner loop

The following plot (Fig. 9) shows the control voltage of the four on/off valves at the slave side. As it can be seen, in free motion as well as under contact, mode 1 (i.e., all valves are closed) and mode 8 (i.e., all valves exhaust) are most used, which allow to keep the difference of pressure in the chambers $\left(P_{p}-P_{n}\right)$ constant. These two modes allow for the chattering and energy consumption to be significantly reduced. Similar result can be observed at the master side.

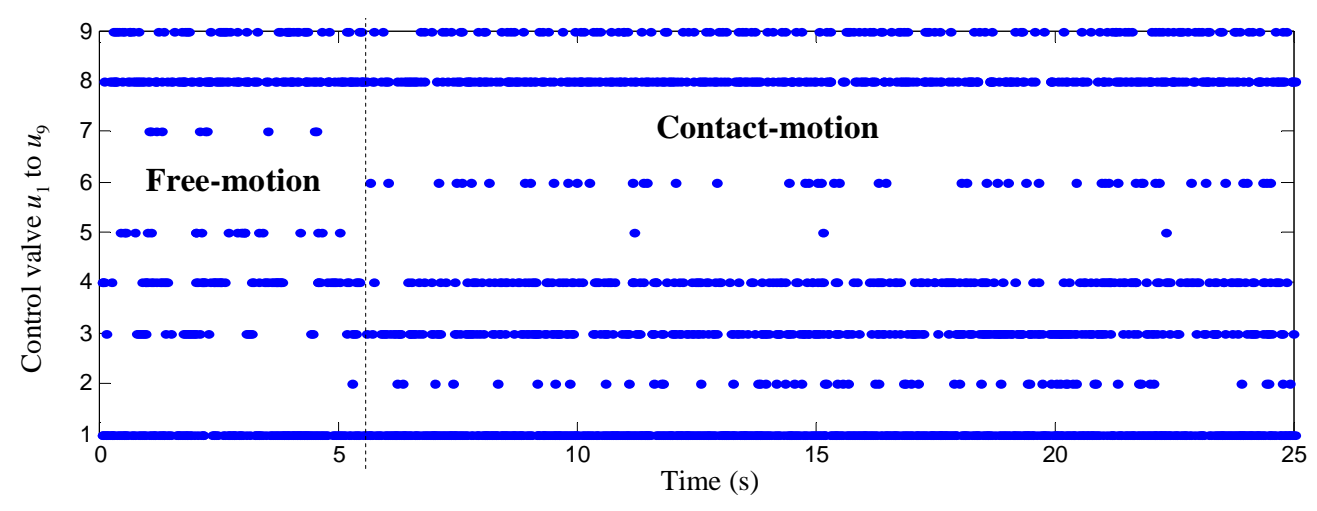

Fig. 9. Evolution of the control value in hybrid algorithm at the slave side

b) Three-channel (3CH) case

Another benefit of the general $4 \mathrm{CH}$ architecture of Fig. 4 is that by proper adjustment of the control parameters, it is possible to obtain two classes of $3 \mathrm{CH}$ control architectures, which can be transparent under ideal conditions [14]. In this way, there is no need for master/operator or slave/environment interaction force measurement. The need for fewer force sensors without degrading transparency makes the $3 \mathrm{CH}$ architectures attractive from the implementation point of view [14].

Fig. 10 shows the master and the slave positions and force tracking profiles for the $3 \mathrm{CH}$ teleoperation system in which $C_{2}$ $=C_{6}+1=1$ and $C_{3}=C_{5}+1=0$. 


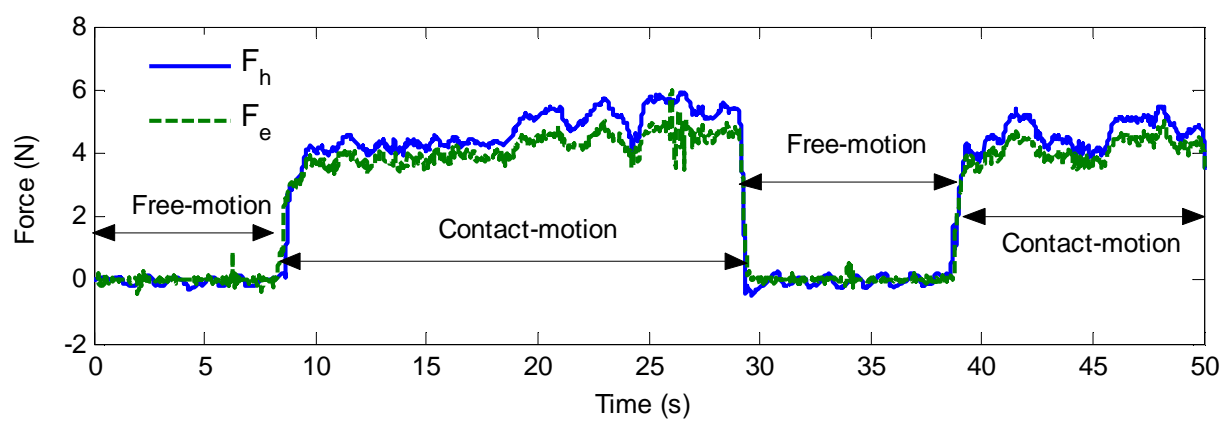

(a). Force tracking

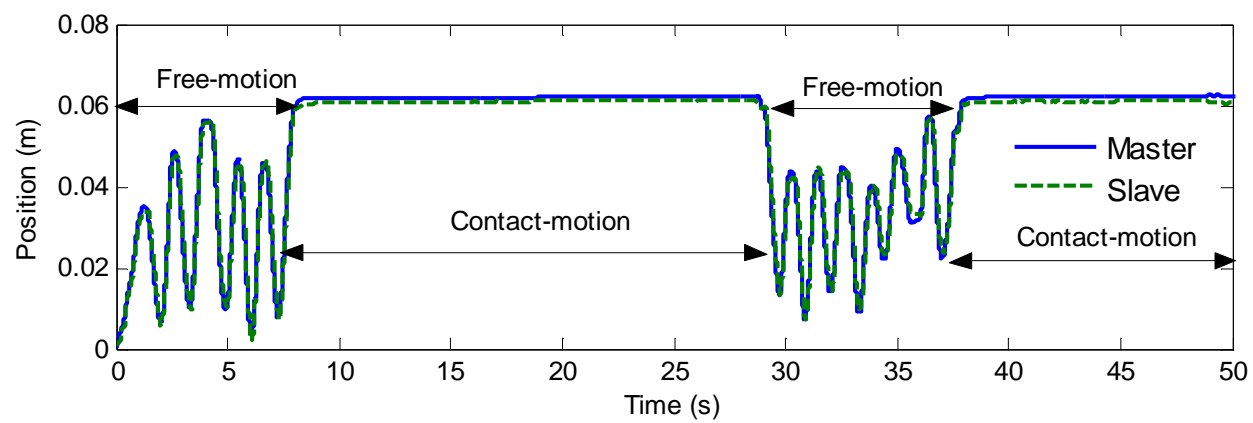

(b). Position tracking

Fig. 10. Transparent performance achieved with the experimental $3 \mathrm{CH}$ teleoperation systems

Note that Fig. 6 and Fig. 10 show similar profiles for the same choice of position controllers $\left(C_{m}\right.$ and $\left.C_{s}\right)$ but for $C_{2}=C_{6}$

$+1=3$ and $C_{3}=C_{5}+1=3(4 \mathrm{CH}$ system $)$, and $C_{2}=\mathrm{C}_{6}+1=1$ and $C_{3}=\mathrm{C}_{5}+1=0$ (3CH system), respectively. The results show that the $4 \mathrm{CH}$ architecture leads to a better force tracking response compared to the $3 \mathrm{CH}$ architecture. As mentioned above, the $3 \mathrm{CH}$ case can theoretically achieve perfect transparency similar to the $4 \mathrm{CH}$ case. However, in practice, due to the lack of master/operator interaction force measurement, the force tracking performance in the $3 \mathrm{CH}$ architecture is somewhat degraded.

To sum up, quantitative assessments of the tracking errors of Fig. 6 and Fig. 10 are provided in the following table:

TABLE II

FORCE AND POSITION TRACKING ERRORS (RMS VALUE) IN STEADY STATE

\begin{tabular}{|c|c|c|c|c|}
\hline & $\begin{array}{c}\text { Force } \\
\text { (free motion) }\end{array}$ & $\begin{array}{c}\text { Force } \\
\text { (contact motion) }\end{array}$ & $\begin{array}{c}\text { Position } \\
\text { (free motion) }\end{array}$ & $\begin{array}{c}\text { Position } \\
\text { (contact motion) }\end{array}$ \\
\hline $4 \mathrm{CH}$ architecture & $0.32 \mathrm{~N}(5.3 \%)$ & $0.2 \mathrm{~N}(3.4 \%)$ & $1.1 \mathrm{~mm}(4.1 \%)$ & $0.4 \mathrm{~mm}(0.7 \%)$ \\
\hline $3 \mathrm{CH}$ architecture & $0.3 \mathrm{~N}(5 \%)$ & $0.7 \mathrm{~N}(12 \%)$ & $1.2 \mathrm{~mm}(4.5 \%)$ & $0.5 \mathrm{~mm}(0.9 \%)$ \\
\hline
\end{tabular}




\section{2) Frequency domain analysis}

To further investigate the teleoperation system transparency, an analysis of the hybrid parameters in the frequency domain is carried out. To do this, we use the hybrid representation of the two-port network model of a master-slave system. A complete discussion of the hybrid matrix analysis can be found in [14], [25]. In this representation,

$$
\left[\begin{array}{c}
F_{h} \\
-Y_{s}
\end{array}\right]=\left[\begin{array}{ll}
h_{11} & h_{12} \\
h_{21} & h_{22}
\end{array}\right]\left[\begin{array}{l}
Y_{m} \\
F_{e}
\end{array}\right]
$$

From (15) and (11) perfect transparency is achieved if and only if the hybrid matrix $H$ has the following form:

$$
H_{\text {ideal }}=\left[\begin{array}{cc}
0 & 1 \\
-1 & 0
\end{array}\right]
$$

Since $F_{e}=0$ in the free-motion test data, the frequency responses $h_{11}=F_{h} /\left.Y_{m}\right|_{F_{e}=0}$ and $h_{21}=-Y_{s} /\left.Y_{m}\right|_{F_{e}=0}$ can be found by applying the spectral analysis function spa of Matlab. Also, by using contact-mode test data, the other two hybrid parameters can be obtained as $h_{12}=F_{h} / F_{e}-h_{11} Y_{m} / F_{e}$ and $h_{22}=-Y_{s} / F_{e}-h_{21} Y_{m} / F_{e}$ [22]. The magnitude of the hybrid parameters of the $3 \mathrm{CH}$ and the $4 \mathrm{CH}$ teleoperation systems are shown in Fig. 11. As it can be seen, $\left|h_{12}\right|$ is above the $0 \mathrm{~dB}$ level for the $3 \mathrm{CH}$ case while it is close to $0 \mathrm{~dB}$ in the $4 \mathrm{CH}$ case. This is in agreement with the time-domain force profiles in Fig. 6 and Fig. 10, where force tracking in $3 \mathrm{CH}$ architecture is not on par with that in the $4 \mathrm{CH}$ case. This, however, does not affect free-space position tracking as illustrated in the $\left|h_{21}\right|$ spectra of Fig. 11. As it can be observed, for both cases $\left|h_{21}\right|$ spectra are close to OdB, showing excellent position tracking for frequencies up to $100 \mathrm{rad} / \mathrm{s}$. As expected from (16), low values of the output admittance $\left(h_{22}\right)$ in the $3 \mathrm{CH}$ and $4 \mathrm{CH}$ architectures show that the slave's movement in response to external force disturbances quickly converges to zero when the master is locked in motion. With regard to $h_{11}$, due to the mass of the handle between the force sensor and the operator's hand, over high frequencies the input impedance $h_{11}=F_{h} / Y_{m}$ is identified less accurately than the other hybrid parameters. Nonetheless, low values of $h_{11}$ over low frequencies are evidence of the fact that when the slave is in free space, the user will not experience a force or sticky feel of free-motion movements in the $3 \mathrm{CH}$ and $4 \mathrm{CH}$ cases, which would have been undesirable. 

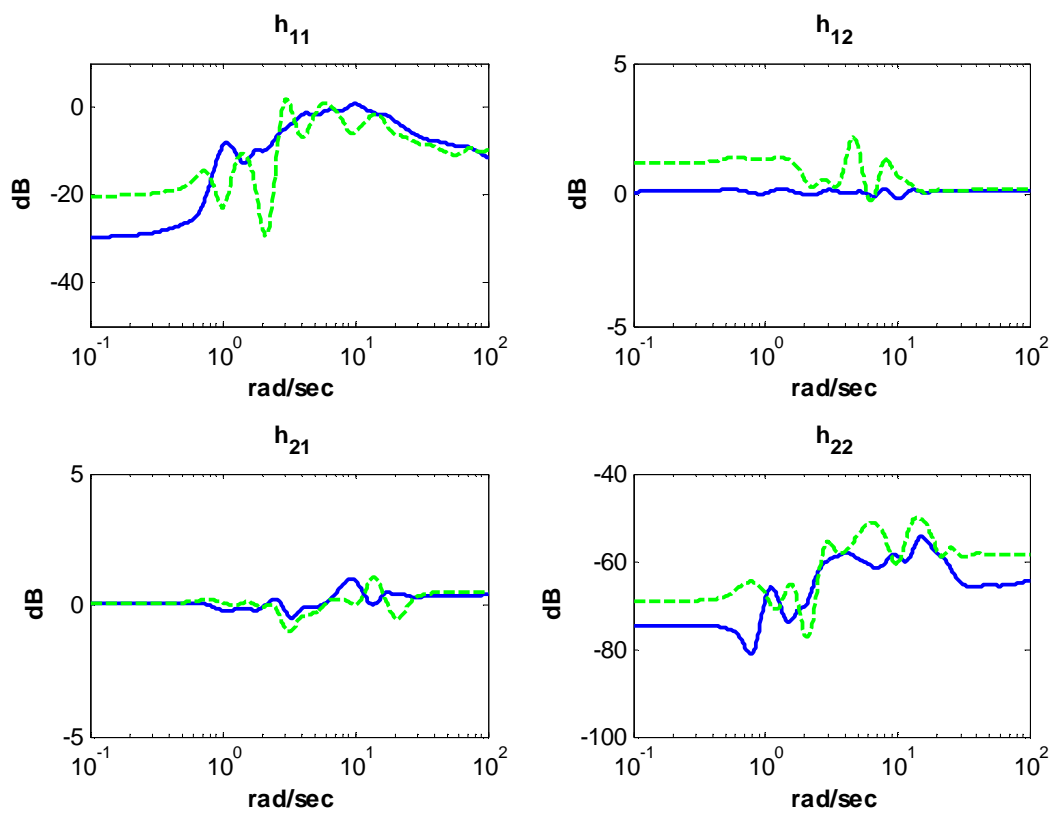

Fig. 11. Frequency spectra of the hybrid parameters for the teleoperation systems. (Solid) 4CH. (Dashed) $3 \mathrm{CH}$.

\section{CONCLUSION}

In this paper, pneumatic actuators with inexpensive solenoid valves were chosen for development of a master-slave teleoperation system. To efficiently control the switching on/off valves in terms of the force output by the piston, a new hybrid algorithm has been successfully developed and implemented in experiments. This technique not only takes into account the non-linear behavior of the mass flow rate but also the switching control of the solenoid valves. The results show that it is possible to achieve transparent teleoperation without using the more costly proportional servo-valves. In experiments, it was observed that with the four-channel bilateral teleoperation control architecture employing this hybrid control algorithms, satisfactory force and position tracking between the master and the slave is obtained under both freemotion and contact-motion conditions.

While the proposed hybrid control in this paper is based on a one-step-ahead prediction of pressures in the pneumatic chambers, an aspect of future work is to extend the control law to involve a multi-step prediction in order to improve the tracking performance. Finally, nonlinear control strategies for pneumatic actuators with solenoid valves such as those in [7], [27] have not been implemented in teleoperation systems yet. This represents an alternative research path to be investigated.

\section{REFERENCES}

[1] H. Kazerroni, «Human-robot interaction via the transfer of power and information signals », IEEE Transactions on Systems, Man and Cybernetics, vol. 20, $\mathrm{n}^{\circ} .2$, p. 450-463, 1990.

[2] P. F. Hokayem and M. W. Spong, « Bilateral teleoperation: An historical survey », Automatica, vol. 42, n . 12, p. 2035-2057, 2006.

[3] N. Yu, C. Hollnagel, A. Blickenstorfer, S. S. Kollias, and R. Riener, « Comparison of MRI-compatible mechatronic systems with hydrodynamic and pneumatic actuation », IEEE/ASME Transactions on Mechatronics, vol. 13, nº. 3, p. 268-277, 2008. 
[4] N. Yu, W. Murr, A. Blickenstorfer, S. Kollias, and R. Riener, «An fMRI compatible haptic interface with pneumatic actuation », in IEEE International Conference on Rehabilitation Robotics (ICORR), 2008, p. 714-720.

[5] K. Tadano and K. Kawashima, «Development of a master slave system with force sensing using pneumatic servo system for laparoscopic surgery », IEEE International Conference on Robotics and Automation, vol. 18, p. 947-952, 2007.

[6] B. Tondu, S. Ippolito, J. Guiochet, and A. Daidie, «A Seven-degrees-of-freedom Robot-arm Driven by Pneumatic Artificial Muscles for Humanoid Robots », The International Journal of Robotics Research, vol. 24, nº. 4, p. 257-274, 2005.

[7] X. Shen, J. Zhang, E. J. Barth, and M. Goldfarb, « Nonlinear averaging applied to the control of pulse width modulated (PWM) pneumatic systems », American Control Conference, vol. 5, p. 4444-4448, 2004.

[8] T. Noritsugu, « Pulse-width modulated feedback force control of a pneumatically powered robot hand », Proceedings of International Symposium of Fluid Control and Measurement, p. 47-52, 1985.

[9] M. Taghizadeh, A. Ghaffari, and F. Najafi, «Improving dynamic performances of PWM-driven servo-pneumatic systems via a novel pneumatic circuit », ISA Transactions, vol. 48, nº. 4, p. 512-518, 2009.

[10] M. Q. Le, M. T. Pham, R. Moreau, and T. Redarce, «Comparison of a PWM and a Hybrid Force Control for a Pneumatic Actuator Using On/off Solenoid Valves », IEEE/ASME International Conference on Advanced Intelligent Mechatronics (AIM), Canada, p. 1146-1151, 2010.

[11] J. M. Retif, X. Lin-Shi, A. M. Llor, and F. Morand, « New hybrid direct-torque control for a winding rotor synchronous machine », IEEE 35th Annual Power Electronics Specialists Conference, vol. 1, p. 1438-1442, 2004

[12] G. Papafotiou, T. Geyer, and M. Morari, « A hybrid model predictive control approach to the direct torque control problem of induction motors », International Journal of Robust and Nonlinear Control, vol. 17, n. 17, p. 1572-1589, 2007.

[13] X. Lin-Shi, F. Morel, A. M. Llor, B. Allard, and J. M. Rétif, «Implementation of hybrid control for motor drives », IEEE Transactions on Industrial Electronics, vol. 54, n ${ }^{\circ} .4$, p. 1946-1952, 2007

[14] M. Tavakoli, A. Aziminejad, R. V. Patel, and M. Moallem, « High-fidelity bilateral teleoperation systems and the effect of multimodal haptics », IEEE Transactions on Systems, Man, and Cybernetics, Part B, vol. 37, nº. 6, p. 1512-1528, 2007.

[15] I. Aliaga, A. Rubio, and E. Sanchez, «Experimental quantitative comparison of different control architectures for master-slave teleoperation », IEEE Transactions on Control Systems Technology, vol. 12, nº. 1, p. 2-11, 2004.

[16] N. Chopra, P. Berestesky, and M. W. Spong, «Bilateral Teleoperation over Unreliable Communication Networks », IEEE Transactions on Control Systems Technology, vol. 16, nº. 2, p. 304-313, 2008

[17] A. Aziminejad, M. Tavakoli, R. V. Patel, and M. Moallem, « Transparent Time-Delayed Bilateral Teleoperation Using Wave Variables », IEEE Transactions on Control Systems Technology, vol. 16, nº 3, p. 548-555, 2008.

[18] B. W. Andersen, The analysis and design of pneumatic systems. Wiley New York, 1967.

[19] R. Blackburn, Shearer, Fluid Power Control. John Wiley and Sons, Inc, 1960.

[20] D. McCloy, « Discharge characteristics of servo valve orifices », Fluid International Conference, p. 43-50, 1968.

[21] M. Q. Le, M. T. Pham, R. Moreau, and T. Redarce, «Transparency of a pneumatic teleoperation system using on/off solenoid valves », IEEE International Conference on Robot and Human Communication (ROMAN), Italy, p. 15-20, 2010.

[22] M. Tavakoli, R. V. Patel, and M. Moallem, Haptics for Teleoperated Surgical Robotic Systems. World Scientific, 2008.

[23] M. Q. Le, M. T. Pham, M. Tavakoli, and R. Moreau, «Development of a hybrid control for a pneumatic teleoperation system using on/off solenoid valves », IEEE International Conference on Intelligent Robots and Systems (IROS), Taiwan, p. 5818-5823, 2010.

[24] D. A. Lawrence, «Stability and transparency in bilateral teleoperation », IEEE Transactions on Robotics and Automation, vol. 9, ${ }^{\circ}$. 5, p. 624637, 1993.

[25] S. E. Salcudean, M. Zhu, W.-H. Zhu, and K. Hashtrudi-Zaad, «Transparent Bilateral Teleoperation under Position and Rate Control», International Journal of Robotics Research, vol. 19, $\mathrm{n}^{\circ} .12$, p. 1185-1202, 2000

[26] K. B. Shimoga, «A survey of perceptual feedback issues in dexterous telemanipulation: Part I. Finger force feedback », Proceedings in IEEE Virtual Reality Annual International Symposium, p. 263-270, 1993.

[27] T. Nguyen, J. Leavitt, F. Jabbari, and J. E. Bobrow, «Accurate sliding-mode control of pneumatic systems using low-cost solenoid valves », IEEE/ASME Transactions on mechatronics, vol. 12, $\mathrm{n}^{\circ} .2$, p. 216-219, 2007. 